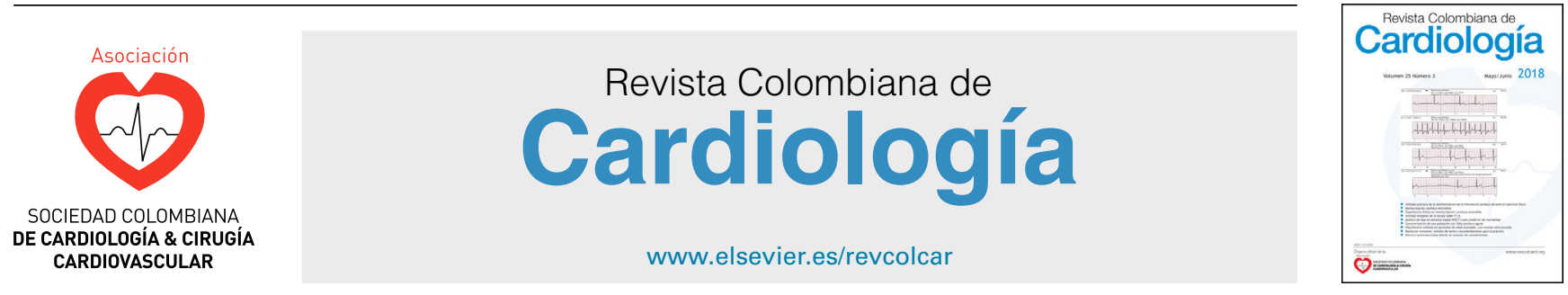

CARDIOLOGÍA DEL ADULTO - ARTÍCULO ORIGINAL

\title{
Abordaje radial distal izquierdo a través de la tabaquera anatómica para la realización de angiografía coronaria
}

\author{
Manuel J. Liévano, Jorge E. Villegas, Jorge de J. Sánchez Cantillo* y Gabriel Acosta
}

Departamento de Hemodinamia y Cardiología Intervencionista, Fundación Universitaria de Ciencias de la Salud - Hospital de San José de Bogotá, Bogotá, Colombia

Recibido el 29 de octubre de 2018; aceptado el 26 de marzo de 2019

Disponible en Internet el 9 de julio de 2019

\author{
PALABRAS CLAVE \\ Angiografía; \\ Cateterismo \\ cardíaco; \\ Arteria radial distal \\ izquierda
}

\begin{abstract}
Resumen
Objetivo: describir la experiencia de un servicio en angiografía coronaria en cuanto al abordaje radial distal izquierdo a través de la tabaquera anatómica.

Material y método: de abril a agosto de 2018 se asignaron 40 pacientes a angiografía coronaria por vía radial izquierda distal a través de la tabaquera anatómica. Se hizo un análisis de tipo descriptivo de las principales variables cuantitativas asociadas al procedimiento (frecuencia de acceso fallido, procedimiento fallido, tiempo de procedimiento, tiempo de fluoroscopia, nivel de dolor en el paciente, sensación de comodidad para el paciente y para el operador, cantidad de medio de contraste empleado y algunas variables demográficas). Se incluyeron pacientes mayores de 18 años con indicación de arteriografía coronaria para estudio diagnóstico y con pulso palpable en la zona de la tabaquera anatómica.

Resultados y conclusión: la cantidad de medio de contraste en promedio empleada fue de $49 \mathrm{ml}$ $(S D \pm 13)$, el tiempo promedio de procedimiento fue de 11,3 minutos $(S D \pm 4.3)$ y el tiempo de fluoroscopia promedio fue de 3,3 minutos $(S D \pm 2.8)$. La mediana para el nivel de dolor en los pacientes fue de 4, la mediana para la comodidad del operador fue de 10 y la mediana para la comodidad en el paciente fue de 10 . Se concluye así que el acceso radial distal izquierdo es una alternativa técnicamente factible, ofrece comodidad para el paciente y el operador, y arroja bajas tasas de falla y complicaciones en pacientes seleccionados.

(C) 2019 Sociedad Colombiana de Cardiología y Cirugía Cardiovascular. Publicado por Elsevier España, S.L.U. Este es un artículo Open Access bajo la licencia CC BY-NC-ND (http:// creativecommons.org/licenses/by-nc-nd/4.0/).
\end{abstract}

\footnotetext{
* Autor para correspondencia.

Correo electrónico: jorgesanchezcantillo@gmail.com (J.J. Sánchez Cantillo).
} 


\section{KEYWORDS}

Angiography;

Cardiac

catheterisation;

Left distal radial

artery

\section{Left distal radius approach through the anatomical snuff box to perform coronary angiography}

\begin{abstract}
Objective: To present the experience of one Department in coronary angiography as regards the left distal radius approach through the anatomical snuff box.

Material and Method: A total of 40 patients were subjected to coronary angiography through the anatomical snuff box, from April to August 2018. A descriptive analysis was performed on the quantitative variables associated with the procedure (frequency of failed access, failed procedure, procedure time, fluoroscope time, patient pain level, feeling of comfort for the patient and for the operator, amount of contrast used, and some demographic variables). The study included patients over 18 years-old with an indication for coronary angiography for a diagnostic study, and with a palpable pulse in the anatomic snuff box area.

Results and conclusion: The mean amount of contrast media employed was $49 \mathrm{ml}(\mathrm{SD} \pm 13)$, the mean procedure time was 11.3 minutes $(S D \pm 4.3)$, and the mean fluoroscope time was 3.3 minutes $(S D \pm 2.8$ ). The median for the pain level in patients was 4 , the median for comfort of the operator was 10 , and the median for comfort of the patient was also 10 . It was able to be concluded that the left distal radial access is a technically feasible alternative. It is comfortable for the patient and the operator, with low failure rates and complications in selected patients. (c) 2019 Sociedad Colombiana de Cardiología y Cirugía Cardiovascular. Published by Elsevier España, S.L.U. This is an open access article under the CC BY-NC-ND license (http:// creativecommons.org/licenses/by-nc-nd/4.0/).
\end{abstract}

\section{Introducción}

Las enfermedades cardiovasculares tienen una prevalencia creciente, hecho que genera alto impacto principalmente en las tasas de mortalidad y de sobrevida de la población general; según la Organización Mundial de la Salud (OMS) 17 millones de personas mueren cada año por esta causa ${ }^{1}$.

Desde el punto de vista asistencial, es importante contar con técnicas innovadoras que permitan precisar el diagnóstico y optimizar el plan terapéutico para los pacientes. La angiografía coronaria y la angioplastia se han convertido en las estrategias de elección para el diagnóstico y tratamiento de la enfermedad coronaria ${ }^{2}$.

El abordaje vascular es un predictor de complicaciones para los pacientes con enfermedad cardiovascular que reciben intervención por vía percutánea. El abordaje de la arteria femoral conlleva un riesgo relativo sustancial de complicaciones hemorrágicas, particularmente relacionadas con el calibre de los catéteres y con el diámetro del introductor arterial, razón por la que se han invertido esfuerzos en la innovación del conocimiento en esta área, cambiando el sitio del abordaje y reduciendo el diámetro vascular para evitar complicaciones, es decir empleando abordajes por arterias más pequeñas como la radial ${ }^{2}$. En este sentido, el acceso vascular para dichos procedimientos ha ccambiado con el tiempo, pasando del acceso vascular frecuente a nivel femoral al acceso vascular radial en el año 2007, con miras a proporcionar al paciente mejores parámetros de seguridad y mayor eficiencia durante su intervención ${ }^{2}$.

Hoy en día hay gran preferencia por el abordaje radial derecho, empleando particularmente dicha lateralidad por ergonomía del operador; no obstante, esta técnica tiene dificultades relacionadas principalmente con la tortuosidad vascular en cercanía a la arteria subclavia derecha, particularidad menos frecuentes en su homónimo anatómico contralateral, lo cual puede llegar a favorecer la manipulación óptima y el avance de los catéteres hasta nivel coronario, evento que acorta el tiempo de procedimiento y, por ende, reduce el tiempo de exposición a fluoroscopia ${ }^{3}$.

Los estudios clínicos llevados a cabo hasta el momento han demostrado reducción de complicaciones con el abordaje radial (en comparación con el acceso femoral), así como de aquellas relacionadas con el sitio de acceso al cateterismo; sin embargo, la información obtenida en la actualidad es escasa en cuanto a los beneficios entre el abordaje radial derecho e izquierdo, pues la evidencia aún es muy heterogénea y poco concluyente, situación que se explica por el abordaje adecuado y por los resultados de los estudios, que pueden ser operador dependientes ${ }^{4}$.

La probabilidad de tortuosidad vascular de la subclavia izquierda es menor respecto a la arteria subclavia derecha, lo cual facilita el acceso a la aorta ascendente durante el procedimiento. De igual forma, desde el punto de vista técnico, el acceso a la arteria mamaria izquierda en los pacientes con revascularización quirúrgica es menos complejo que en quienes se accede por vía radial derecha ${ }^{5}$.

De otra parte, el abordaje radial izquierdo poco se usa en la práctica clínica, debido a las dificultades que se presentan al realizar el acceso vascular en la región anterior del antebrazo, es menos ergonómico para el operador, en especial con los pacientes obesos, es incómodo para el paciente debido a la posición en abducción en la que debe permanecer el miembro superior izquierdo, requiere más cercanía con los rayos de fluoroscopia y muchas veces más tiempo de radiación; adicionalmente existe un alto subregistro de los datos del procedimiento, lo cual dificulta mucho más su análisis. Después de explorar o intentar el acceso por la vía radial derecha, si este es fallido, usualmente se puede 
considerar el acceso radial izquierdo convencional o el acceso vascular por la vía femoral; sin embargo, este acceso radial izquierdo en la región anterior o en la región distal por la tabaquera anatómica es ventajoso por cuanto hay menor tortuosidad y es posible canular con mayor facilidad el puente mamario izquierdo en el caso de los pacientes que han sido revascularizados de forma quirúrgica ${ }^{6-8}$.

Una de las dificultades del acceso radial izquierdo convencional, se deriva de la posición que deben adoptar el paciente y el operador; mediante el acceso radial distal por la tabaquera anatómica, esta dificultad mejora significativamente dado que el paciente no tendría que abducir el brazo izquierdo e incluso podría apoyarlo sobre su abdomen; adicionalmente, el operador no deberá flexionar la parte superior de su cuerpo sobre el paciente para realizar el procedimiento. En cuanto a las complicaciones vasculares y de sangrado, se sabe que el acceso radial aporta un alto perfil de favorabilidad en comparación con el acceso femoral en todos los escenarios ${ }^{9-13}$.

Existen varias series de casos, los cuales muestran resultados alentadores para el acceso radial izquierdo por la tabaquera anatómica, planteando una excelente alternativa o incluso pudiendo considerarse como la opción inicial en los pacientes que serán sometidos a cateterismo cardíaco izquierdo ${ }^{4,14-17}$.

\section{Objetivos}

Describir la experiencia en la práctica de angiografía coronaria diagnóstica mediante el abordaje radial izquierdo por la tabaquera anatómica.

\section{Métodos}

Estudio observacional, descriptivo, tipo serie de casos, en el que se practicó acceso radial izquierdo a 40 pacientes ambulatorios referidos para angiografía coronaria diagnóstica, desde abril hasta agosto de 2018, en tres instituciones de la ciudad de Bogotá (Colombia) (Hospital de San José, Hospital Universitario Clínica San Rafael y Clínica Los Nogales).

Se incluyeron pacientes mayores de 18 años con indicación de arteriografía coronaria para estudio diagnóstico y pacientes con pulso palpable en la zona de la tabaquera anatómica.

Se excluyeron aquellos con alteraciones arteriales documentadas o irregularidad en el test de Allen y pacientes con historia conocida de cateterismo fallido por la vía radial anterior izquierda.

Antes de efectuar la intervención se obtuvo consentimiento informado escrito. Una vez verificado el pulso adecuado en la tabaquera anatómica por palpación manual, con el antebrazo izquierdo en ligera abducción y flexionado sobre el abdomen y ligera flexión distal de la mano, se procedió a la infiltración de $1 \mathrm{ml}$ de lidocaína al $2 \%$ y luego se puncionó la arteria con una aguja (21G) con una inclinación entre $35^{\circ}-45^{\circ}$ grados, dirigida hacia el sitio donde el pulso se palpa con mayor intensidad. Una vez se logró el acceso, confirmado por la presencia de flujo adecuado de retorno de sangre arterial, se avanzó una guía 0.018 manteniendo ligeramente flexionado el antebrazo. Posteriormente, se avanzaron introductores 5 y 6 Fr hidrofílicos y no hidrofílicos; con miras a disminuir el riesgo de vasoespasmo y oclusión de la arteria radial, se administraron 200 mcg de nitroglicerina intraarterial y posteriormente 70-100 UI por kilo de heparina no fraccionada intravenosa. Luego de retirar el introductor se hizo hemostasia con dispositivo TR Band (fig. 1).

Se analizaron variables cuantitativas consideradas relevantes para el estudio (acceso fallido, procedimiento fallido, tiempo del procedimiento, tiempo de fluoroscopia, ergonomía para el operador, nivel de dolor en el paciente, sensación de comodidad para el paciente, cantidad de medio de contraste). Las variables categóricas se reportaron como frecuencias absolutas y relativas y las variables cuantitativas como media y desviación estándar (tablas 1 y 2 ).

Los datos fueron recolectados con un formulario electrónico en los teléfonos inteligentes que alimentaron la base de datos de Google Drive, datos que posteriormente se incorporaron en una tabla de Excel y a los cuales se les hizo un análisis estadístico con el software STATA 13.

Se utilizó el consentimiento informado de cada institución previo a la angiografía coronaria.

El protocolo de investigación fue presentado y aprobado por el Comité de Ética en investigación en Humanos del Hospital San José y las Clínicas San Rafael y Los Nogales.

\section{Resultados}

De 40 pacientes que se sometieron al acceso radial izquierdo distal por la tabaquera anatómica, 25 eran de sexo masculino $(62,5 \%)$, con edad promedio de 63,9 años (desviación estándar 11.2 años, edad mínima de 33 años y máxima de 82 años). A 12 pacientes (30\%) se les había realizado cateterismo cardíaco izquierdo previo, 10 de ellos por la vía radial anterior lo cual fue útil al momento de analizar las variables de dolor y ergonomía. 36 pacientes (90\%) eran ambulatorios; en 1 paciente $(0,4 \%)$ el acceso vascular fue fallido, pero en todos se logró realizar el procedimiento angiográfico. En 3 (7,5\%) pacientes se identificó hematoma en la tabaquera anatómica y la mediana del dolor valorado con escala análoga numérica fue de 4 (fig. 2). La cantidad de medio de contraste en promedio empleada fue de $49 \mathrm{ml}$ $(D E \pm 13)$; el tiempo promedio de procedimiento fue de 11,3 minutos $(D E \pm 4,3)$ y el tiempo de fluoroscopia promedio fue de 3,3 minutos $(D E \pm 2,8)$.

La percepción de ergonomía por el paciente y por el operador, así como el nivel de dolor para el paciente durante el procedimiento fueron evaluados mediante el uso de una tabla visual análoga, con rangos desde 1 hasta 10 , donde 1 es el menor valor (menos cómodo/menos doloroso) y 10 el máximo (máxima comodidad/máximo dolor); la mediana para el nivel de dolor en los pacientes fue de 4, la mediana para la comodidad del operador fue de 10 y la mediana para la comodidad en el paciente fue de 10 (fig. 3).

Ningún paciente ambulatorio requirió de hospitalización ni hubo complicaciones vasculares o neurales posteriores al procedimiento. No fue necesario emplear catéteres más largos que $100 \mathrm{~cm}$ y la dificultad para manipular catéteres solo se presentó en 3 pacientes en los cuales se notó que por la mayor edad, los tejidos en la tabaquera mostraban mayor rigidez y, por tanto, mayor resistencia.

En las tablas 1 y 2 se resumen las características sociodemográficas y procedimentales. 


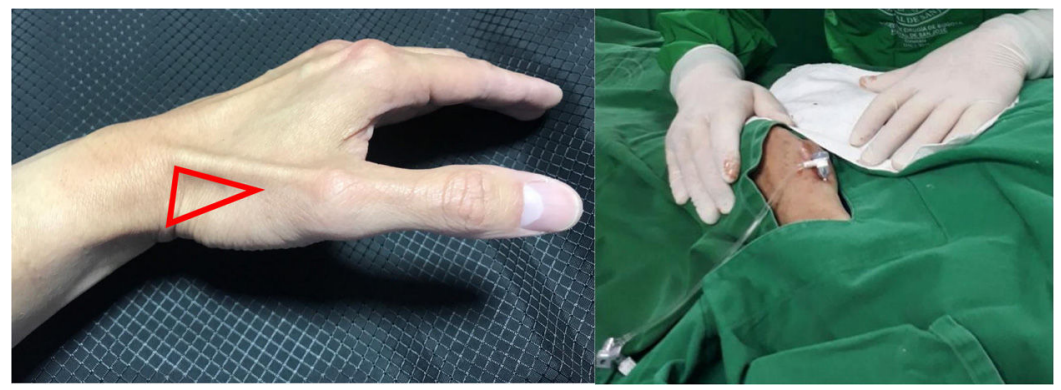

Figura 1 Acceso radial distal por la tabaquera anatómica ${ }^{19}$.

Tabla 1 Características sociodemográficas y clínicas

\begin{tabular}{ll}
\hline Variables & $\mathrm{n}=40$ \\
\hline Edad media años $( \pm D E)$ & $63,9( \pm 11,2)$ \\
Sexo masculino $n(\%)$ & $25(62,5)$ \\
Ambulatorio $n(\%)$ & $36(90)$ \\
Antecedentes & \\
$\quad$ Hipertensión arterial $\mathrm{n}(\%)$ & $29(72,5)$ \\
$\quad$ Diabetes mellitus $\mathrm{n}(\%)$ & $11(27,5)$ \\
$\quad$ Falla cardiaca sistólica FEVI $<50 \% \mathrm{n}(\%)$ & $6(15)$ \\
Infarto de miocardio previo $\mathrm{n}(\%)$ & $9(22,5)$ \\
$\quad$ Enfermedad renal crónica (TFG $<60$ & $33(82,5)$ \\
$\mathrm{ml} /$ min) $\mathrm{n}(\%)$ & \\
$\quad$ Angiografía coronaria previa $\mathrm{n}(\%)$ & $12(30)$ \\
\hline
\end{tabular}

Tabla 2 Características del procedimiento

\begin{tabular}{ll}
\hline Variables & $\mathrm{n}=40$ \\
\hline Acceso vascular fallido $\mathrm{n}(\%)$ & $1(0,4)$ \\
Procedimiento fallido $\mathrm{n}(\%)$ & $0(0)$ \\
Tiempo de procedimiento promedio en min & 11,3 \\
Tiempo de fluoroscopia promedio en min & $3,3( \pm 2,8)$ \\
Medio de contraste promedio en $\mathrm{ml}$ & $49( \pm 13)$ \\
Dificultad de manipulación de catéteres $\mathrm{n}(\%)$ & $3(7,5)$ \\
Hematoma & $3(7,5)$ \\
Tortuosidad vascular $\mathrm{n} \%$ & $3(7,5)$ \\
Espasmo radial $\mathrm{n} \%$ & $5(12,5)$ \\
Dificultad para avanzar catéteres en la & $3(7,5)$ \\
tabaquera $\mathrm{n} \%$ & \\
Necesidad de un catéter $>100 \mathrm{~cm} \mathrm{n} \%$ & $0(0)$ \\
\hline
\end{tabular}

\section{Discusión}

La vía de acceso radial izquierdo distal por la tabaquera anatómica es útil y práctica para la realización de angiografía coronaria, requiere de entrenamiento y una curva de aprendizaje si el operador se encuentra entrenado en cateterismo por la vía radial anterior convencional ${ }^{4}$; adicionalmente, es una muy buena alternativa cuando no es posible abordar al paciente por la vía radial derecha o incluso puede ser la vía de acceso a considerar como inicial por las ventajas en cuanto a hemostasia, menor grado de invalidez las siguientes 24 horas dado el mayor número de pacientes diestros en la población general y las menores tasas de complicaciones vasculares y neurales documentadas. También es ideal para la angiografía coronaria en los pacientes que han tenido

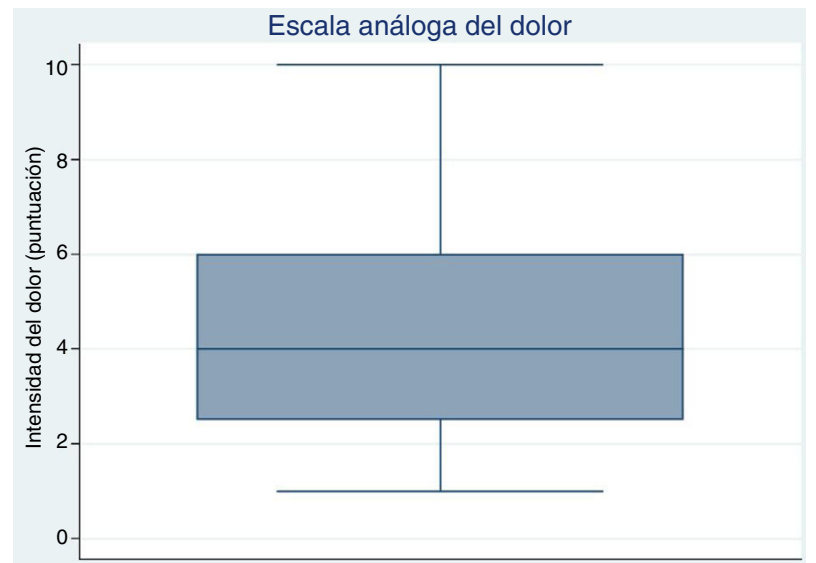

Figura 2 Dolor por escala visual análoga (EVA)

Diagrama de cajas y bigotes de la percepción de la intensidad del dolor, que muestra una mediana del nivel del dolor de 4, valorada con una escala visual análoga numérica.

revascularización quirúrgica con puente mamario izquierdo dada la facilidad para canularlo debido al acceso por la arteria subclavia izquierda ${ }^{5}$.

El acceso de la arteria radial distal en la tabaquera anatómica por vía derecha o izquierda en otros estudios se ha consolidado como opción atractiva y factible tanto para los pacientes como para los operadores ${ }^{16}$.

Incluso el uso de esta vía en un metaanálisis reciente sugiere una diferencia pequeña pero estadísticamente significativa en términos de uso de medio de contraste y tiempo de fluoroscopia a favor de los procedimientos coronarios hechos mediante abordaje radial izquierdo en comparación con el abordaje radial derecho, sin ninguna diferencia significativa en el sitio de acceso y otras complicaciones del procedimiento entre los dos enfoques, variables analizadas en este estudio, que evidencia esta tendencia ${ }^{4}$.

Este estudio tiene como limitaciones una muestra poblacional pequeña y su carácter netamente descriptivo. Se requieren más estudios para determinar si esta técnica aporta mayor comodidad al paciente y la posible preservación de la arteria radial para ser considerada con mayor frecuencia.

La mayor fortaleza de este estudio es la evaluación de un gran número de variables que permiten observar la tendencia a favor del uso de la vía de acceso radial izquierdo por la tabaquera anatómica para la práctica de angiografía coronaria. 


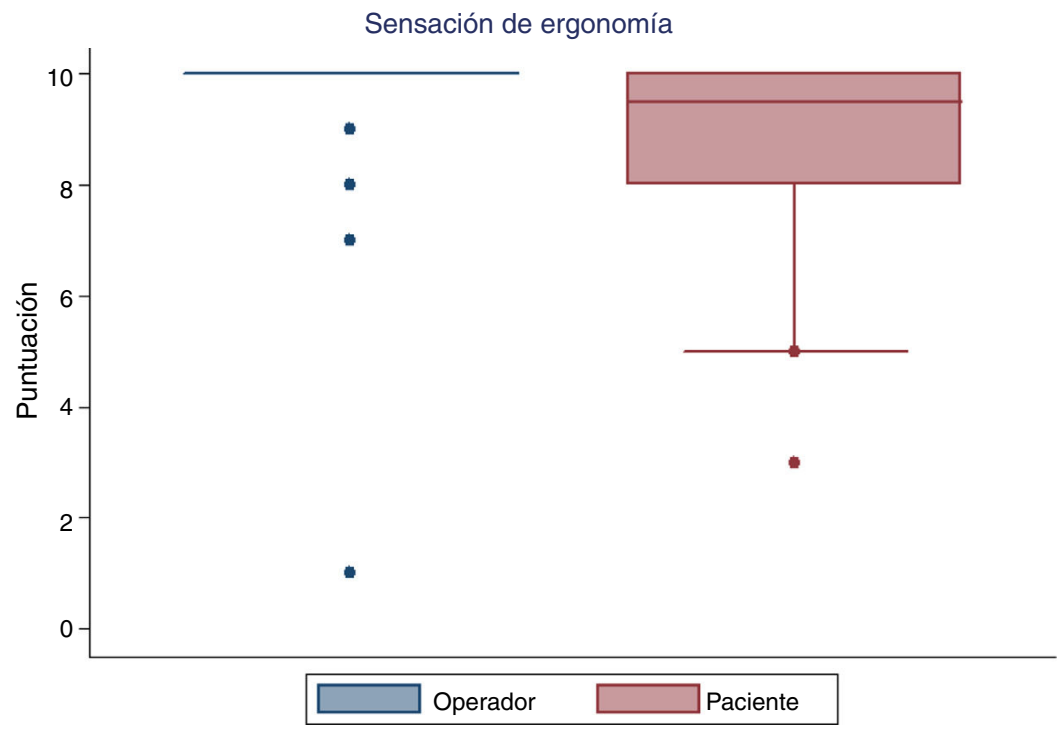

Figura 3 Percepción de ergonomía durante el procedimiento por el operador y por el paciente.

Diagrama de cajas y bigotes de la percepción de la ergonomía por el paciente y por el operador, evaluada con una escala visual análoga. Se halló una mediana de 10 para comodidad del operador y una mediana de 10 de comodidad del paciente.

\section{Conclusiones}

La vía de acceso radial izquierdo distal por la tabaquera anatómica es útil y práctica para la realización de angiografía coronaria, y se convierte en una opción atractiva y factible tanto para los pacientes como para los operadores, con tiempos de fluoroscopia y cantidad de medio de contraste utilizada similar a la de otras vías de acceso vascular.

\section{Financiación}

Ninguna.

\section{Conflictos de interés}

Ninguno.

\section{Bibliografía}

1. Organización WH. ¿Qué son las enfermedades cardiovasculares? http://www.who.int/cardiovascular_diseases/about_cvd/es/ Organización Mundial de la Salud; 2011. [citado 2017].

2. Kiemeneij F, Laarman GJ. Abordaje percutáneo de la arteria transradial para la implantación de un stent Palmaz-Schatz coronario. Am Heart J. 1994;128:167-74.

3. Freixa X, Trilla M, Feldman M, Jimnez M, Betriu A, Masotti $M$. Abordaje transradial derecho versus izquierdo para el cateterismo coronario en pacientes octogenarios. Cateterización e Intervenciones Cardiovasculares. 2012;80:267-72.

4. Shah RM, Patel D, Abbate A, Cowley MJ, Jovin ES. Comparación de los procedimientos coronarios transradiales a través del abordaje de la arteria radial derecha frente a la radial izquierda: un metanálisis. Cateterización e Intervenciones Cardiovasculares. 2016;88:1027-33.
5. Campeau L. Abordaje percutáneo de la arteria radial para la angiografía coronaria. Cateterización y Diagnóstico Cardiovascular. 1989;16:3-7.

6. Kiemeneij F, Laarman GJ, Odekerken D, Slagboom TA. Una comparación aleatoria de la angioplastia coronaria transluminal percutánea mediante abordajes radiales, braquiales y femorales: estudio de acceso. Am Coll Cardiol. 1997;29:1269-75.

7. Pristipino C, Pelliccia F, Granatelli A, Pasceri V, Roncella A, Speciale G, et al. Comparación de las complicaciones hemorrágicas relacionadas con el acceso en mujeres versus hombres sometidos a cateterización coronaria percutánea mediante la arteria radial frente a la femoral. Revista Americana de Cardiología. 2007;99:1216-21.

8. Jolly SS, Yusuf S, Cairns J, Niemel. Acceso radial versus femoral para la angiografía coronaria e intervención en pacientes con síndromes coronarios agudos (RIVAL): un ensayo aleatorizado, de grupos paralelos, multicéntrico. The Lancet. 2011;377:1409-20.

9. Valgimigli M, Gagnor A, Calabr. Acceso radial versus femoral en pacientes con síndromes coronarios agudos sometidos a tratamiento invasivo: un ensayo multicéntrico aleatorizado. The Lancet. 2015;385:2465-76.

10. Hamon M, Filippi-Codaccioni E, Riddell JW, Lepage O. Impacto pronóstico del sangrado mayor en pacientes con síndromes coronarios agudos Una revisión sistemática y un metanálisis. Eurolntervención. 2007;3:400-8.

11. Valgimigli M, Saia F, Guastaroba P, Menozzi A, Magnavacchi P, Santarelli A, et al. Intervención transradial versus transfemoral para el infarto agudo de miocardio: un análisis de puntuación de propensión ajustado y emparejado del registro multicéntrico REAL (registro regionale angioplastiche dell'emilia-romagna). JACC: Intervenciones Cardiovasculares. 2012;5:23-35.

12. Sciahbasi A, Romagnoli E, Burzotta F, Trani C, Sarandrea A, Summaria F, et al. Enfoque transradial (izquierda vs. derecha) y tiempos de procedimiento durante los procedimientos coronarios percutáneos: estudio TALENT. Am Heart J. 2011;161: 172-9.

13. Cerda A, del Sol M. Anatomical Snuffbox y su significado clínico. Una revisión de la literatura. Int J Morphol. 2015;33:1355-60. 
14. Kiemeneij F. Acceso transradial distal izquierdo en la caja de rapé anatómica para angiografía coronaria (IdTRA) e intervenciones (IdTRI). Eurolntervención. 2017;13:851-7.

15. Soydan E, Ak"n M. Angiografía coronaria utilizando el abordaje radial distal izquierdo: un lugar alternativo a la angiografía coronaria radial convencional. Anatol J Cardiol. 2018;19: 243-8.

16. Valsecchi O, Vassileva A, Cereda AF, Canova P, Satogami K, Fiocca L, et al. Experiencia clínica temprana con acceso transradial distal derecho e izquierdo en la caja de rapé anatómica en 52 pacientes consecutivos. J Cardiol invasor. 2018;30: 218-23.

17. Sgueglia GA, Di Giorgio A, Gaspardone A, Babunashvili A. Bases anatómicas y fundamentos fisiológicos del acceso a la arteria radial distal para procedimientos percutáneos coronarios y endovasculares. JACC Cardiovasc Interv. 2018;11:2113-9. 